Economía Agraria y Recursos Naturales. ISSN: 1578-0732. Vol. 6, 11. (2006). pp. 139-156

\title{
Los factores determinantes del paso de las explotaciones de la leche a la carne en la Cornisa Cantábrica: análisis empírico para una comarca del interior de Galicia
}

\author{
Alfonso Ribas Álvarez*, Edelmiro López Iglesias* y María L. Loureiro**
}

\begin{abstract}
RESUMEN: Este trabajo pretende contribuir al estudio de los factores que determinan el paso de las explotaciones de vacuno de leche a vacuno de carne en la Cornisa Cantábrica, examinando para ello la relación de este proceso con las características productivas y familiares de las explotaciones. Además, se contrasta en concreto el papel jugado por la concentración parcelaria, la principal medida de reforma de estructuras agrarias aplicada en la agricultura de estas regiones durante las últimas décadas. Los datos empleados se refieren al período 1985-1999 y a una muestra de 458 explotaciones con ganado bovino (activas en 1999) sobre una población de 1.420 unidades productivas situadas en la Comarca Interior de la provincia de A Coruña. De esas unidades productivas, 46 dejaron de producir leche para reconvertirse a carne en el período estudiado, manteniéndose el resto dedicadas a la producción láctea durante todo el período. Los resultados indican que el abandono de la leche está relacionado de forma positiva con la edad del titular, mientras que otras variables que afectan al proceso de forma negativa son: la superficie total de
\end{abstract}

Este trabajo se ha beneficiado del apoyo de los proyectos de investigación: «Cambio estructural y políticas agrarias: el caso de los sistemas agrarios especializados en cultivos herbáceos, olivar y ganadería bovina» (CAMESPA), financiado por el Ministerio de Ciencia y Tecnología (referencia AGL20012680-C02-02); y «Cambio estructural e políticas agrarias», financiado por la Dirección Xeral de Investigación e Desenvolvemento de la Xunta de Galicia (código PGIDIT02PXIC24201PN). Los autores agradecen también los comentarios hechos por dos revisores anónimos.

* Alfonso Ribas y Edelmiro López son profesores del Departamento de Economía Aplicada de la Universidad de Santiago.

** María L. Loureiro es investigadora Ramón y Cajal en el Instituto de Estudos e Desenvolvemento Económico de Galicia (IDEGA)-Universidad de Santiago.

Dirigir correspondencia a: Alfonso Ribas Álvarez. Departamento de Economía Aplicada. Escola Politécnica Superior. Campus Universitario.27002 Lugo. E-mail: idgribas@usc.es

Recibido en octubre de 2005. Aceptado en octubre de 2006. 
la explotación, la producción media por vaca y la superficie media por parcela al inicio del período, la existencia de sucesión y el número de personas de la familia que trabajan fuera de la explotación. En cambio, el hecho de haberse realizado la concentración parcelaria no tiene influencia directa en el proceso de abandono de la producción lechera.

PALABRAS CLAVE: Concentración parcelaria, Explotaciones bovinas, Reorientación productiva, Regresión logística.

Clasificación JEL: Q12, Q18.

\title{
Analysis of the determinant factors affecting changes from dairy to cattle production in the Cantabrian Coast: Empirical analysis for an inland area of Galicia.
}

\begin{abstract}
SUMMARY: This paper analyzes the factors that contribute to changes in farming specialization, and it assesses the importance of such factors in the occurred transformations from dairy to cattle farms in Northern Spain. In line with this objective, we examine the role played on this process by the farms' characteristics as well as farmers' socio-economic factors. Furthermore, we asses the role played by public land consolidation policies, which were the main structural policies applied in this area during the last decades. Our data came from a representative sample of 458 livestock farms (active in 1999) in the province of A Coruña, during the period 1985-1999. From the total, 46 farms switched their productive orientation quitting dairy and entering into cattle production during the period of study. The rest kept their dairy specialization. Results indicate that dairy exit is positively related to farmer's age. At the same time, it is negatively related to the following variables: dairy production per cow, total land, the existence of heirs, and the amount of family members working outside the farm. Furthermore, the fact that the farm had been involved in a land consolidation process did not have any direct effect on this change of productive orientation.
\end{abstract}

KEYWORDS: Dairy farms, Cattle production, Land consolidation, Logistic regression.

JEL classification: Q12, Q18.

\section{Introducción}

Las producciones bovinas conforman la base económica del sector agrario de las Comunidades Autónomas de la Cornisa Cantábrica, aportando el 53\% del valor de su producción agraria, de la cual $2 / 3$ corresponden a la leche y $1 / 3$ a la carne y ganado vacuno. Esta dependencia de los productos bovinos es especialmente acusada en Asturias y Cantabria (78\% de la producción agraria en ambas regiones), mientras que en Galicia el porcentaje desciende al 44\%, muy superior en cualquier caso al 10,7\% que suponen en el conjunto de España (MAPA, 2005).

Acorde con lo anterior, las explotaciones con ganado bovino constituyen el núcleo de la agricultura de la Cornisa, tanto en términos sociales y económicos como en lo que respecta a la gestión del territorio. Concretamente, con datos del Censo Agrario de 1999 y limitándonos a las «unidades profesionales» ${ }^{1}$, las explotaciones bovinas

${ }^{1}$ El universo de explotaciones considerado en las Encuestas sobre la Estructura de las Explotaciones Agrarias. 
suponen el $68 \%$ del total, aportan el $80 \%$ del Margen Bruto (MBT) y poseen el $62 \%$ de la SAU.

En Galicia, al igual que en el conjunto de España, las explotaciones de bovino han experimentado un fuerte ajuste estructural desde la integración en la UE. Este proceso ha tenido su núcleo en la acelerada desaparición de explotaciones lácteas, que se ha correspondido en buena medida con un abandono definitivo de la actividad pero en parte también con una reconversión hacia la carne, fenómeno éste común al constatado en el resto de la Cornisa Cantábrica (Alberdi, 2001).

En el caso de Galicia, de acuerdo con los datos de la Enquisa de Explotacións de Vacún del Instituto Galego de Estatística (IGE), entre 1993 y 2001 el número de explotaciones que sólo cuentan con vacas de ordeño («lácteas puras») se redujo en un $77,2 \%$, en tanto que aumentó ligeramente la cifra de aquéllas que sólo tienen vacas de carne y se duplicó la de explotaciones mixtas. Como consecuencia, las explotaciones «lácteas puras» pasaron de representar el 65,9\% del total de explotaciones bovinas en 1993 al 24,9\% en 2001, mientras que las «cárnicas puras» (sólo con vacas de carne) aumentaron del 26,0\% al 47,4\% (CIEF-IDEGA, varios años).

La intensa desaparición de explotaciones lecheras se acompañó de una moderada reducción del número de vacas de ordeño, en tanto aumentaban las que no se ordeñan. Pero lo más destacable es el contraste en la dinámica y características estructurales de ambos sectores: en el lácteo la acelerada desaparición de unidades productivas se acompañó de un fuerte incremento de su tamaño medio (en el período 1993-2001 pasó de 6,8 a 19,4 vacas/ explotación); mientras que las explotaciones cárnicas se caracterizan por mantener una dimensión media muy inferior y casi estancada $(4,0 \mathrm{y}$ 5,5 vacas/ explotación, respectivamente).

Esa redistribución de las explotaciones según su especialización y de las vacas según su aptitud, más que a un proceso de reorientación cárnica y un dinamismo de esta rama, parece responder principalmente al abandono de la producción láctea, acelerado por la existencia del sistema de cuotas (CIEF-IDEGA, varios años). Alberdi (2001) llega a las mismas conclusiones para el País Vasco.

Todos los datos apuntan a que el vacuno de carne se fue configurando en Galicia desde los años 70 , continuando esto en los 90, principalmente como una producción para explotaciones marginales, expulsadas del sector lácteo, que por su reducido tamaño sobreviven en la mayor parte de los casos gracias a otras fuentes de ingresos ${ }^{2}$. Partiendo de ese diagnóstico, una de las cuestiones fundamentales es si el fenómeno de salida del sector lácteo y de reconversión a la carne constituye una situación estable, o solamente un paso intermedio en el camino hacia el abandono definitivo de la actividad agraria.

El propósito de este trabajo es contribuir a precisar esta cuestión, mediante el análisis de las características productivas y familiares de las explotaciones que abandonan la producción lechera para pasar al vacuno de carne. Además, se pretende con-

\footnotetext{
${ }^{2}$ De hecho, según nuestras estimaciones a partir de los datos del Censo Agrario de 1999, el 52\% de la renta total de estas explotaciones procedía de otras actividades y de pensiones, frente a sólo un $24 \%$ en las explotaciones que tenían exclusivamente vacas de leche. La metodología de este cálculo puede verse en Sineiro et al. (2005).
} 
trastar si las políticas de reforma de estructuras como la concentración parcelaria (de especial relevancia en Galicia) influyen en ese proceso. Los datos utilizados en este estudio se recogieron a través de una encuesta a titulares de explotaciones con ganado bovino de la Comarca Interior de la provincia de A Coruña, y tienen la ventaja de que permiten seguir la evolución de estas unidades productivas durante un período amplio de tiempo (1985-1999), que coincide con los años de reorientación cárnica de muchas explotaciones lácteas gallegas ${ }^{3}$.

El interés de nuestro estudio se ve reforzado por el hecho de que, a pesar de la extensión del fenómeno en toda la Cornisa Cantábrica, existen muy pocos trabajos de investigación sobre las motivaciones de los titulares para abandonar la producción láctea y pasarse a la carne. Los análisis realizados hasta ahora son en general descriptivos (Alberdi, 2001) y hacen poco hincapié en los factores que influyen en la toma de decisiones por parte de los ganaderos, aspecto que consideramos de gran relevancia.

Este trabajo se estructura del siguiente modo: comenzamos haciendo un repaso de la bibliografía, centrándonos en los factores que influyen en el abandono de la actividad agraria; posteriormente se explica la metodología utilizada; el siguiente apartado se dedica a la exposición y discusión de los resultados obtenidos; para resumir finalmente las principales conclusiones.

\section{Factores que influyen en el abandono de la actividad agraria}

Si aceptamos, como hipótesis de partida, que el paso de las explotaciones de leche a carne constituye una etapa intermedia en el abandono definitivo de la actividad agraria, cabe pensar que los factores que influyen en este proceso son similares a los que desencadenan las salidas definitivas. De hecho, con frecuencia estas salidas constituyen un proceso gradual, en el que los titulares reducen su actividad, a veces trabajando a tiempo parcial fuera de la explotación o simplemente cambiando a una actividad menos exigente en trabajo, para abandonar después definitivamente. Pensamos que esto último es lo que sucede en muchas ocasiones en las explotaciones que dejan el sector lácteo en Galicia (López Garrido, 1993; López Iglesias, 1998 y Sineiro et al., 2005).

Las características de las explotaciones que cambian de la actividad lechera a la producción de carne no están muy estudiadas, pero sí existen numerosos estudios sobre las características productivas y familiares de las explotaciones que cesan en la actividad agraria (Kimhi et al., 1999; Weiss, 1999; Stilbauer et al., 2000; Goetz et al., 2001; Glauben et al., 2003a y 2003b; López Iglesias, 1996). En la literatura se han establecido principalmente cuatro factores desencadenantes de ese cese: el tamaño de las explotaciones, la edad del titular, la carencia de sucesor y la presencia de un empleo extra-agrario - pluriactividad-.

Los análisis apuntan por lo general a una relación inversa entre el tamaño de la explotación y la probabilidad de cesar la actividad (Weiss, 1996; Kimhi et al., 1999;

\footnotetext{
${ }^{3}$ Los detalles de la encuesta aparecen especificados en Ribas, A. (2004).
} 
Stiglbauer et al., 2000), que se vincularía con la existencia de rendimientos crecientes a escala. Aunque no faltan los autores que destacan la mayor facilidad de las pequeñas explotaciones para sobrevivir en periodos de descenso de la demanda (Lieberman, 1990) o la tenacidad de las mismas a la hora de sobrevivir (Gasson et al., 1995). Baur (2000), en su estudio para la explotaciones suizas, encuentra que la probabilidad de abandonar primero decrece con el aumento del tamaño para posteriormente elevarse; esto se debe a que las explotaciones de gran dimensión dependen mucho de las tierras arrendadas, lo que hace dificultosa y a la vez arriesgada la sucesión en estas unidades productivas.

El predominio en la agricultura de las formas de producción familiares en casi todos los países desarrollados, y la consiguiente transmisión de las explotaciones en el seno de la familia, hace que la sucesión cobre una importancia vital en el estudio del abandono (López Iglesias, 1996) ${ }^{4}$. Entre los autores que en los últimos años han analizado la sucesión y su relación con el abandono está Alberdi (2001), que para el País Vasco concluye que cuando los titulares alcanzan los 50 años y la explotación carece de relevo familiar ésta sufre un proceso de estancamiento y progresiva reducción de la actividad. En esta línea, el citado autor observa en concreto que la falta de sucesión conduce al cambio de la leche a la carne, como un paso en el proceso de desaparición. Kimhi (1994) examina las transferencias de explotaciones en Israel, concluyendo que el momento de transmitir la propiedad de las mismas varía con las características de la explotación y la familia. Kimhi et al. (1999) estudian la importancia de la sucesión en los planes de jubilación de los agricultores, concluyendo que las consideraciones sobre ambas cuestiones en la familia agraria son inseparables. Stilgbauer et al. (2000) analizan, para el caso de Austria, las características de las familias y las explotaciones que influyen en la sucesión y el abandono, concluyendo que el tamaño de la explotación y la diversificación productiva inciden positivamente en la sucesión, así como también lo hacen el tamaño de la familia y la educación del titular. Glauben et al. (2002) concluyen en la misma línea que las explotaciones más grandes y especializadas tienen más posibilidades de contar con sucesión dentro de la familia. Respecto a la edad del titular y la sucesión, concluyen que la relación no es lineal y que la probabilidad de tenerla primero aumenta con la edad y después decrece. La relación negativa entre edad y probabilidad de sucesión para los titulares de edades muy avanzadas sugiere que los agricultores que posponen la sucesión lo hacen precisamente por las dificultades para encontrar un sucesor dentro de la familia.

Es de esperar que las probabilidades de que una explotación abandone aumenten con la edad del titular, especialmente cuando no existe sucesión. Jackson-Smith et al. (2000) concluyen que el factor más importante asociado con el cierre de explotaciones lecheras en Wisconsin es la edad del titular, siendo los abandonos especialmente altos en el grupo de titulares mayores de 55 años. Alberdi (2001) señala que la causa principal que empuja a la interrupción de esta actividad es la edad del jefe de la explotación. Este, una vez superados los 60 años, se orienta hacia producciones más extensivas.

${ }^{4}$ En Galicia el 97\% de las explotaciones censadas en 1999 tenían como titular una persona física, porcentaje que ascendía al $99 \%$ en las que contaban con ganado bovino. 
Además del tamaño de la explotación, la edad del titular y la sucesión, para estudiar por qué se producen las salidas del sector agrario es necesario analizar la influencia de la pluriactividad (el empleo del titular u otros miembros de la familia en otras actividades). Su efecto sobre las salidas se ha debatido mucho, viéndose en ocasiones como un medio de estabilizar los ingresos de las familias agrarias en períodos de crisis, lo que contribuiría a la supervivencia de la explotación, y en otros casos en cambio como un paso intermedio en el abandono definitivo de la agricultura.

De hecho, en los últimos años numerosos estudios han intentado resolver esta cuestión. Kimhi et al. (1999) y Kimhi (2000) concluyen que las probabilidades de abandonar decrecen con la extensión del empleo extra-agrario, tanto en Israel como en Canadá. En la misma línea, Glauben et al. (2003b) encuentran que en Alemania las regiones con altos porcentajes de agricultores a tiempo parcial tienen menores tasas de abandono, lo que sugiere que el trabajo extra-agrario tiene un impacto estabilizador en las estructuras agrarias. Pfeffer (1989) sin embargo sugiere lo contrario, concluyendo que en Alemania los agricultores a tiempo parcial tienen menores expectativas de continuar con la explotación en el futuro. Weiss $(1997,1999)$, en un sentido similar, encuentra efectos positivos de la existencia de empleo extra-agrario en las probabilidades de abandonar. Swidinsky et al. (1998), en un estudio sobre el empleo extra-agrario en Canadá, concluyen que la tasa de salidas entre los que trabajan a tiempo parcial supera a la de los que lo hacen a tiempo completo. Por otra parte, Stilbauer et al. (2000) estiman que en Austria los titulares que trabajan a tiempo parcial tienen una mayor probabilidad de abandonar la agricultura; lo que según ellos sugiere que el empleo extra-agrario es el primer paso de la explotación familiar en el camino de salida de la actividad agraria. Jackson-Smith et al. (2000) concluyen que la participación del titular o su esposa en el empleo extra-agrario incrementa las posibilidades de que una explotación abandone el sector lácteo.

Por último, algunos autores han estudiado el efecto de algunas medidas de política agraria, como los planes de abandono de la producción láctea, el cese anticipado de la actividad agraria o la concentración parcelaria en el abandono de la agricultura. Gale (1990) concluye que la participación en los programas de abandono sólo es interesante para los titulares de edad avanzada que no planean transferir la explotación a otros miembros de la familia, y para los granjeros menos experimentados y productivos. El nivel de participación sugiere que los efectos a largo plazo del programa en la oferta de leche son pequeños. Además muchas de las explotaciones que dejaron la leche a través del programa habrían probablemente abandonado el sector en cualquier caso. Lo que indica que el programa paga por salidas de explotaciones que hubieran tenido lugar de igual manera. Paniagua (2000) señala que en Castilla y León las explotaciones que se acogen al cese anticipado son pequeñas o muy pequeñas para la media regional (14,9 ha), y no tienen en su mayoría solucionada la sucesión.

En las dos últimas décadas existen varios trabajos que analizan la aplicación de la política de Concentración Parcelaria en España (Liss, 1987) y en diversas Comunidades Autónomas como los de De la Riba (1990) para Aragón; Alário (1991) para Castilla León; Fernández García et al. (1992a; 1992b) para Asturias, De los Ríos (1996) para la Comunidad de Madrid o Crecente et al. (2002) para Galicia. Estos trabajos son dispares tanto en su extensión y profundidad con la que abordan el tema como en 
su enfoque. Sus conclusiones acerca de los resultados de la concentración están muy marcadas por la heterogeneidad existente entre las agriculturas y territorios que cada uno de ellos analiza.

Ninguno de ellos analiza en profundidad la relación entre concentración parcelaria y abandono de la actividad lechera o de la actividad agraria en general. Los pocos que hacen referencia a ello, constatan una relación inversa entre concentración parcelaria y abandono, es decir, la concentración actúa como un freno al abandono. Sin embargo, en la mayoría de los casos se trata de aseveraciones o impresiones de los autores, que no están contrastadas con datos empíricos que permitan cuantificar la intensidad con que estos elementos se relacionan. Van Huylenbroek et al. (1996) han señalado que la concentración parcelaria, al mejorar la base productiva, facilitar el trabajo y aumentar la productividad, influye positivamente en la viabilidad de las explotaciones a medio plazo, llevando a una menor tasa de abandono. Crecente et al. (2002) encuentran una asociación positiva - en Galicia - entre la realización de la concentración parcelaria y el mantenimiento de población en el medio rural.

El presente estudio pretende contribuir a esta literatura en dos aspectos fundamentales. Por un lado, determinar para el caso español - y en concreto para la Cornisa Cantábrica - cuáles son las características productivas y familiares de las explotaciones que están abandonando el sector lácteo para dedicarse a las vacas de carne. Por otro, se pretende analizar el efecto que ha tenido en ese proceso la política de concentración parcelaria, principal medida aplicada en España para la mejora de las estructuras agrarias desde la década de 1950.

\section{Metodología}

\section{Datos}

La muestra se compone de 458 explotaciones con ganado bovino, que se seleccionaron en 1999 entre el total de las que tenían al menos una cabeza de vacuno en el Censo de Saneamiento de 1998. Todas las explotaciones seleccionadas producían leche en 1985; de ellas 46 habían abandonado esa producción por la de carne en el período 1985-1999, continuando las 412 restantes dedicadas a la leche al final del período. Los titulares fueron entrevistados durante el año 1999, recogiéndose información acerca de las características productivas y familiares de la explotación ${ }^{5}$. Las entrevistas a los titulares fueron realizadas por personal técnico de AFRICOR (Asociación Frisona para el Control de Rendimientos) en las propias explotaciones.

Las explotaciones están localizadas en la Comarca Interior de la provincia de A Coruña (MAPA, 1978), provincia que en 1998 era la de mayor producción lechera de España (MAPA, 2000). La comarca está compuesta por 24 ayuntamientos, con una fuerte especialización en la producción láctea; de hecho, en ellos se concentra el $48 \%$ del censo bovino de la provincia y el $50 \%$ de la cuota láctea.

\footnotetext{
${ }^{5}$ El cuestionario utilizado para la realización de la encuesta está disponible, por parte de los autores, para todos aquellos lectores interesados.
} 
El objetivo fundamental de la entrevista era proporcionar una información precisa y fiable de las principales características estructurales y socioeconómicas de las explotaciones de vacuno concentradas y no concentradas, así como pulsar la opinión de los titulares de las mismas sobre la política de concentración parcelaria.

La unidad básica de la encuesta es la explotación de vacuno y la población objeto de estudio es el conjunto de explotaciones de vacuno de las 28 parroquias seleccionadas en los 24 ayuntamientos de la comarca. A efectos de muestreo, y del proceso posterior de estimación y cálculo de factores de ponderación, la población se dividió en 5 estratos de tamaño en función del número de vacas reproductoras de la explotación: 1-4, 5-9, 10-19, 20-44 y mayores de 44 vacas. Cada uno de estos estratos constituyó una población independiente a la hora del muestreo.

Para la selección de la muestra se aplicó un diseño estratificado aleatorio con Afijación Optima de Neyman para un intervalo de confianza del 95\% y un error de estimación del $4 \%$ a nivel de estrato, siendo la muestra representativa para el conjunto de la Comarca (Neyman, 1934). Se fijó el tamaño mínimo de muestra en 120 encuestas, distribuidas por estrato como se puede ver en la tabla 1. A pesar de ello se realizaron un mayor número de encuestas, consistiendo la muestra para el presente estudio en 448 explotaciones.

TABLA 1

Tabla de error de estimación relativo

\begin{tabular}{|c|c|c|c|}
\hline & $\begin{array}{c}\text { N. }{ }^{o} \text { total de } \\
\text { explotaciones } \\
\text { en la población }\end{array}$ & $\begin{array}{l}\text { N. }{ }^{\circ} \text { total de explotaciones } \\
\text { a entrevistar para un error de } \\
\text { estimación relativo del } 4 \%\end{array}$ & $\begin{array}{l}\text { N. }{ }^{\circ} \text { de explotaciones } \\
\text { en la muestra }\end{array}$ \\
\hline 1. ${ }^{\text {er }}$ Estrato (1-4 vacas) & 336 & 9 & 26 \\
\hline 2. ${ }^{\circ}$ Estrato (5-9 vacas) & 387 & 14 & 46 \\
\hline $3{ }^{\text {er }}$ Estrato $=(10-19$ vacas $)$ & 408 & 29 & 140 \\
\hline $4^{\circ}$ Estrato (20-44 vacas) & 246 & 37 & 208 \\
\hline 5. ${ }^{\circ}$ Estrato (> 44 vacas) & 43 & 31 & 38 \\
\hline TOTAL & 1.420 & 120 & 458 \\
\hline
\end{tabular}

Una vez definido el tamaño de la muestra para cada estrato, se procedió a seleccionar las explotaciones a entrevistar por medio de un muestreo sistemático con arranque aleatorio a partir del Censo de Saneamiento Ganadero de 1998.

Tomando como base estudios previos, se seleccionaron una serie de variables independientes para construir un modelo de regresión logística múltiple referido al abandono de la producción láctea. Para su cómputo se utilizó el procedimiento PROC LOGISTIC (SAS, 1995) que permitió obtener los estimadores de máxima verosimilitud de los coeficientes de la regresión, los odds ratio (OR) y los intervalos de confianza al $95 \%(95 \% \mathrm{CI})$.

En la tabla 2 figuran las variables utilizadas en la estimación del modelo de regresión logística junto a sus definiciones y los signos esperados para las mismas. La variable dependiente es la variable abandono, que viene determinada con un valor 1 si 
la explotación abandonó la producción láctea en el periodo estudiado y con valor 0 si en 1999 continuaba dedicándose a la misma. Las variables independientes son la variable dummy o indicadora de si se ejecutó la concentración parcelaria $(\mathrm{CON})$, la superficie total de la explotación en hectáreas en 1985 (SUPTO85), el número de vacas por hectárea de superficie total de la explotación en 1985 (VCSUPTO85), la producción media por vaca en 1985 (PLVAC85), la superficie media de las parcelas de la explotación en 1985 en hectáreas (SMPT85), la edad del titular en 1999 (IDATITU99), la existencia o no de sucesión asegurada en este año (SUC99) y el número de personas de la unidad familiar que trabajan fuera de la explotación en 1999 (FORA99) (ver tabla 2).

TABLA 2

Descripción de las variables usadas en el modelo de regresión logística y signo esperado de las mismas

\begin{tabular}{|c|c|c|}
\hline Variable & Descripción & Signo esperado \\
\hline ABANDONO & $0=$ Continua en el sector lácteo & \\
\hline & 1= Abandona el sector lácteo durante el periodo y se pasa a carne & \\
\hline $\mathrm{CON}$ & $\begin{array}{l}\text { Variable dummy con valor } 1 \text { si la explotación está concentrada o } 0 \text { si } \\
\text { no lo está }\end{array}$ & - \\
\hline SUPTO85 & Superficie total de la explotación en 1985 (has) & - \\
\hline VCSUPTO85 & Número de vacas por hectárea de superficie total en 1985 & + \\
\hline PLVAC85 & Producción de leche por vaca en 1985 (litros) & - \\
\hline SMPT85 & Superficie media de las parcelas de la explotación en 1985 (has) & - \\
\hline IDATITU99 & $\begin{array}{l}\text { Variable dummy con valor } 1 \text { si el titular tiene más de } 50 \text { años o } 0 \text { en } \\
\text { caso contrario. }\end{array}$ & + \\
\hline SUC99 & $\begin{array}{l}\text { Variable dummy con valor } 1 \mathrm{si} \text { el titular tiene la sucesión asegurada, } 0 \mathrm{si} \\
\text { no tiene sucesión o no la tiene asegurada }\end{array}$ & - \\
\hline FORA99 & $\begin{array}{l}\text { Número de personas de la unidad familiar que trabajan fuera de la } \\
\text { explotación en } 1999\end{array}$ & $?$ \\
\hline
\end{tabular}

En el cuestionario se incluía una pregunta acerca de si la explotación estaba o no concentrada, además de preguntar el año de finalización de las actuaciones. Todas las explotaciones concentradas lo estaban antes de 1980, por lo que el tiempo transcurrido permitiría observar los efectos de esta política sobre el abandono.

La variable SUPTO85 indica el número de hectáreas de las que disponía la explotación en 1985, suponemos que las unidades de mayor tamaño obtienen mayores ingresos, por lo que cabe esperar que la probabilidad de que abandonen la actividad sea menor. La variable VCSUPTO85 se define como el número de animales por hectárea de superficie total de la explotación (carga ganadera) al inicio del periodo. Esta nos da una idea del grado de intensificación de la producción (en este sentido, se espera que las unidades más intensivas tengan menores tasas de abandono). La variable PLVAC85 nos indica la productividad por vaca, aquellas con mayores productividades por vaca serán más competitivas. Se espera que las menos competitivas en 1985 abandonasen antes la actividad y cambiasen de actividad. La variable SMPT85 se define como el tamaño medio de las parcelas de las que disponía la explotación en 
1985. Suponemos las unidades con parcelas de mayor tamaño ven facilitada la mecanización de los trabajos consiguiendo unas productividades por hectárea y trabajo más elevadas, por lo que cabe esperar que será menor su probabilidad de abandonar.

Siguiendo estudios previos, incluimos entre las variables explicativas la edad del titular para controlar los efectos del «ciclo de vida» ${ }^{6}$. La edad del titular en 1999 fue transformada en una variable dummy con valor 1 si era mayor de 50 años y valor 0 si no alcanzaba ese umbral. Se espera que el efecto de esta variable sea positivo sobre el abandono. Al respecto cabe señalar que diversos trabajos han señalado que el efecto de la edad sobre el abandono es negativo para los agricultores más jóvenes, volviéndose positivo cuando alcanzan una edad avanzada. Goetz et al. (2002) proponen como edad en la que los efectos comienzan a influir positivamente en el abandono los 48 años, Weiss (1996) señala una edad de 43 años y Stilbauer et al. (2000) los 38 años.

Para medir las perspectivas de sucesión se preguntó a los titulares sobre quién les sucedería en la explotación, codificándose las respuestas con valor 1 si tenían un sucesor designado y 0 en el caso de que no lo supieran o no tuvieran sucesor. Si la explotación tiene sucesor es previsible que existan menores probabilidades de abandonar, como muestran los estudios precedentes (por ejemplo Kimhi et al., 1999; Glauben et al., 2002; López Iglesias, 1996).

El número de personas que viven en la explotación y trabajan fuera de la misma es una variable que pretende medir el efecto del trabajo extra-agrario en el abandono de la producción láctea. Como ya hemos indicado, el efecto del trabajo extra-agrario sobre el abandono es difícil de prever a priori: por un lado podría pensarse que mayor número de personas trabajando fuera significa menor trabajo en la explotación y menores costes a asumir en el tránsito hacia el abandono definitivo de la agricultura; pero eso podría tener el efecto contrario y suponer un ingreso extra que permitiese el mantenimiento de la explotación a medio plazo.

\section{El modelo}

El modelo analiza cuáles son los determinantes que influyen en el abandono de la producción láctea y el paso a carne por parte de una explotación. Considerándose dos estados posibles para una explotación: o bien produce leche o bien ha abandonado esta producción y se ha pasado a la carne.

En el modelo, se supone que la variable de respuesta, el abandono, puede ser definida según la siguiente relación:

$$
y_{i}^{*}=\beta^{\prime} X_{i}+\varepsilon_{i}
$$

donde $y_{i}^{*}$ es una variable no observable o latente, que se corresponde con la utilidad derivada de cada una de las acciones citadas. Los que se observan son sus resultados (cambio de orientación o no), que pueden ser definidos como:

${ }^{6}$ La hipótesis del «ciclo de vida» establece que el ciclo de vida de la explotación está muy relacionado con la edad del titular de la misma, y consiste en una serie de fases consecutivas de entrada, crecimiento, declive y transferencia o salida. 


$$
\begin{aligned}
& y_{i}=1 \text { si } y_{i}^{*}>0 \\
& y_{i}=0 \text { en caso contrario }
\end{aligned}
$$

$\beta \prime X_{i}$ es un vector que representa el valor esperado de dadas las características $X_{i}$.

De [1] y [2], se obtiene que la probabilidad de abandonar la producción de leche y pasarse a carne es:

$$
P\left(y_{i}=1\right)=P\left(\beta^{\prime} X_{i}+\varepsilon_{i}>0\right)=P\left(\varepsilon_{i}>-\beta^{\prime} X_{i}\right)
$$

Dado que la distribución logística ${ }^{7}$ es simétrica, satisface la siguiente relación:

$$
P\left(y_{i}=1\right)=P\left(\varepsilon_{i}>-\beta^{\prime} X_{i}\right)=F\left(\beta^{\prime} X_{i}\right)
$$

Por lo tanto, la probabilidad de continuar en el sector lácteo puede expresarse como:

$$
P\left(y_{i}=0\right)=1-\mathrm{P}\left(y_{i}=1\right)=F\left(\beta^{\prime} X_{i}\right)
$$

Donde la forma funcional de $F(\cdot)$ depende de los supuestos que se hagan sobre los errores en [1]. En este caso, el modelo logístico puede especificarse como:

$$
P_{i}\left(y_{i}=1\right)=F\left(\beta^{\prime} X_{i}\right)=\frac{1}{1+e^{-\beta^{\prime} X i}}
$$

donde $P_{i}$ es la probabilidad que la i-ésima explotación abandone el sector dado el vector $X_{i}$, de características familiares y productivas de la explotación. Empleando la ecuación (6) el ratio de probabilidades de abandonar sobre no abandonar, viene dado por:

$$
e^{\beta^{\prime} X_{i}}=\frac{P_{i}\left(y_{i}=1\right)}{1-P_{i}\left(y_{i}=1\right)}
$$

Finalmente, haciendo una transformación logarítmica en [7] surge que:

$$
\ln \frac{P_{i}\left(y_{i}=1\right)}{1-P_{i}\left(y_{i}=1\right)}=\beta^{\prime} X_{i}
$$

La variable dependiente en la ecuación [8] es el logaritmo natural del ratio de probabilidades de que cierta acción tenga lugar. En esta ecuación los coeficientes logísticos $\beta$ se pueden interpretar como el cambio en el logaritmo de la probabilidad (log-odds) asociado a un cambio unitario en la variable independiente.

\footnotetext{
${ }^{7}$ La distribución logística tiene una forma que se asemeja a una distribución normal excepto en las colas que son más altas en la distribución logística. Ésta se parece más a una distribución $t$ con 7 grados de libertad (Greene, 2003).
} 
Dado que es más fácil pensar en términos de probabilidades (odds) que de $\log$ $o d d s$, esta forma de interpretar los resultados se adoptará a lo largo del trabajo. Si las odds son mayores de 1 esto indica que la probabilidad de abandonar crece al aumentar en una unidad la variable dependiente. Y tienen el significado del cambio en la probabilidad de abandonar al variar en una unidad la variable dependiente permaneciendo las demás constantes. Los $\beta$ son los coeficientes de regresión que indican la dirección del impacto de la variable sobre la $P\left(y_{i}=1\right)$. Si $\beta$ es positivo (negativo), el factor de cambio será mayor (menor) que 1, lo que implica que los odds aumentan (disminuyen) cuando $x_{i}$ sufre un cambio unitario. En el caso de que el $\beta$ sea nulo, el factor será igual a la unidad, por lo que los odds no varían cuando cambia la correspondiente variable independiente.

\section{Resultados y discusión}

En la tabla 3 figuran los valores medios de las variables seleccionadas, para el conjunto de la muestra y cada uno de los dos grupos estudiados: explotaciones que pasaron de la leche a la carne (cambiaron de orientación productiva) y explotaciones lecheras que continúan en el sector (no cambiaron de orientación productiva). De los resultados del test $\mathrm{T}$ se extrae que existen diferencias estadísticamente significativas al 1 por mil en la media de ambos grupos para todas las variables incluidas en el modelo, excepto para la variable SMPT85 que lo es al 3 por mil y la variable FORA99 (no significativa).

\section{TABLA 3}

Resumen de las variables usadas en el modelo de regresión logística y prueba $\mathbf{T}$ para igualdad de medias entre explotaciones que cambian de leche a carne y explotaciones que continúan en el sector lácteo en el período 1985-1999

\begin{tabular}{|c|c|c|c|c|c|c|c|c|}
\hline \multirow[b]{3}{*}{ Variable } & \multirow{2}{*}{\multicolumn{2}{|c|}{$\begin{array}{c}\text { Todas } \\
\mathrm{N}=\mathbf{4 5 8}\end{array}$}} & \multirow{2}{*}{\multicolumn{2}{|c|}{$\begin{array}{c}\begin{array}{l}\text { No cambiaron } \\
\text { de orientación }\end{array} \\
N=412\end{array}$}} & \multirow{2}{*}{\multicolumn{2}{|c|}{$\begin{array}{c}\begin{array}{c}\text { Cambiaron } \\
\text { de orientación }\end{array} \\
\mathrm{N}=46\end{array}$}} & \multirow{3}{*}{$\begin{array}{c}\text { T- } \\
\text { estadístico }\end{array}$} & \multirow{3}{*}{$P>t$} \\
\hline & & & & & & & & \\
\hline & MEDIA & DESV STD & MEDIA & DESV STD & MEDIA & DESV STD & & \\
\hline $\mathrm{CON}$ & 0,5633 & 0,4965 & 0,5583 & 0,4972 & 0,6087 & 0,4934 & $-0,657$ & 0,514 \\
\hline SUPTO85 & 14,717 & 11,808 & 15,511 & 11,941 & 7,6098 & 7,513 & 6,299 & 0,000 \\
\hline VCSUPTO85 & 1,1989 & 0,7832 & 1,1386 & 0,6488 & 1,7393 & 1,4329 & $-5,065$ & 0,000 \\
\hline PLVAC85 & $3.002,00$ & $1.352,20$ & $3.084,20$ & $1.312,40$ & $2.266,00$ & $1.489,80$ & 3,573 & 0,001 \\
\hline SMPT85 & 1,0086 & 1,0504 & 1,0572 & 12,003 & 0,5738 & 0,4413 & 2,986 & 0,003 \\
\hline IDATITU99 & 0,4716 & 0,4997 & 0,4272 & 0,4953 & 0,8696 & 0,3405 & $-5,901$ & 0,000 \\
\hline SUC99 & 0,2511 & 0,4341 & 0,2767 & 0,4479 & 0,0217 & 0,1474 & 3,834 & 0,000 \\
\hline FORA99 & 0,452 & 0,7764 & 0,4587 & 0,7741 & 0,3913 & 0,8022 & 0,543 & 0,590 \\
\hline
\end{tabular}

Las explotaciones que cambiaron de orientación productiva tenían una superficie total en 1985 notablemente inferior a las que continuaron en el sector lácteo (7,6 y 15,5 has, respectivamente). El Test tipo $\mathrm{T}$ (tabla 3 ) nos indica que existen diferencias 
significativas entre los dos grupos para la variable superficie total de la explotación en 1985 ( $t=6,29$ y $p=<, 0001)$. Asimismo, de los resultados podemos extraer que las unidades productivas que abandonaron la leche tenían en 1985 una mayor carga ganadera (vacas por hectárea de superficie) que las que continúan en el sector, 1,7 y 1,1 respectivamente, pero una producción de leche por vaca notablemente inferior (2.266 y 3.084 litros, respectivamente). De los resultados del Test tipo $\mathrm{T}$ se concluye que existen diferencias significativas entre los dos grupos en las variables relacionadas con la carga ganadera (intensificación) y la producción por vaca (productividad) $(t=-$ 5,06 у $p=0,000$ y $t=3,57$ y $p=0,001$, respectivamente).

Asimismo, las unidades productivas que abandonaron la leche tenían también unas parcelas de superficie notablemente inferior ( 0,57 y 1,05 has, respectivamente). El test tipo T nos muestra que existen diferencias significativas entre los dos grupos en la variable tamaño de la parcela en 1985 ( $t=2,98$ y $p=0,003$, respectivamente).

La media de la variable «edad del titular» (tal como se define en este trabajo) para el conjunto de las explotaciones con bovino censadas en Galicia en 1999 era de 0,64, siendo la media para la muestra de 0,47 . Además, esa edad es significativamente más alta en el grupo de los que abandonan $(0,86)$ que para los que continúan en la producción láctea $(0,42)$. Esto confirma la tendencia de muchos titulares de edad avanzada a orientar sus explotaciones hacia producciones más extensivas, con menores requerimientos de trabajo, especialmente cuando carecen de sucesión dentro de la unidad familiar.

El porcentaje de titulares que declaran contar con sucesión asegurada se sitúa en nuestra muestra en el $24 \%$, siendo esta proporción mucho mayor en aquellas explotaciones que continúan en la actividad lechera (26\%) que en las que se pasaron a carne - donde es prácticamente nula- $(2 \%)$. El número medio de personas con otra actividad es de 0,45 , alcanzando un valor ligeramente superior en las explotaciones que continúan en la producción de leche $(0,45$ y 0,39 , respectivamente).

Existen diferencias significativas en las variables anteriores: edad del titular $(t=$ $-5,91$ y $p=0,000)$ y presencia de sucesor $(t=3,83$ y $p=0,000)$. En cambio, las diferencias no son significativas ni en la variable concentración parcelaria ni en la referida al número de personas que trabajan en otra actividad. El número de personas trabajando fuera, que refleja la pluriactividad de la familia, no varía entre los dos grupos. El diseño del cuestionario no permite separar los casos en los que la actividad externa corresponde al titular, lo que constituye una limitación al trabajo. De hecho, según los datos del Censo Agrario de 1999 para el total de Galicia, el número de titulares con otra actividad lucrativa es ligeramente superior en el grupo de explotaciones de carne (12\%) que en el grupo de explotaciones lecheras (8\%).

En la tabla 4 se muestran los resultados de la regresión logística. Las variables CON y VCSUPTO85 no son estadísticamente significativas. En cuanto al resto de las variables incluidas en el modelo, los signos de los coeficientes coinciden en general con los esperados. Las variables SUPTO85 y PLVAC85 tienen signo negativo como era de esperar, lo que confirma la hipótesis de que un mayor tamaño y especialización al inicio del período reduce las posibilidades de abandonar. De hecho, han sido, las explotaciones pequeñas (con escasa capacidad productiva) y poco modernizadas (baja productividad del rebaño) las que han abandonado en mayor proporción la acti- 
vidad lechera en Galicia en los últimos años. Su déficit de tamaño y tecnología no les ha permitido adaptarse con éxito a las exigencias a las que se enfrenta el sector desde la incorporación de España a la Unión Europea ${ }^{8}$. En otras palabras, las explotaciones que abandonaron la leche, lo hicieron, en parte, por la incapacidad de aumentar su dimensión hasta un tamaño económicamente viable ${ }^{9}$. La variable SMPT85 muestra un signo negativo como era de esperar, lo que confirma la hipótesis de que un mayor tamaño de las parcelas al inicio del período reduce las probabilidades de abandonar. A su vez, la variable SUC99 tiene una influencia negativa en las probabilidades de abandonar el sector lácteo. La variable IDATITU99 cumple también la previsión influyendo positivamente en el paso de la leche a la carne. Con respecto a la variable FORA99, cuya influencia no conocíamos a priori, su signo es negativo. Este resultado coincide con el obtenido en otros trabajos (Kimhi et al., 1999; Baur, 2000), y podría explicarse por la estabilidad que da el ingreso extra-agrario, que permite que las explotaciones se mantengan durante más tiempo en la actividad.

La significación individual de los parámetros se puede ver a través del estadístico asintótico Wald chi-cuadrado. Si el valor de p asociado con cada una de las variables es $<0,1$ quiere decir que las variables son predictores significativos del abandono de la producción láctea. Pues bien, con la excepciones de las variables CON y VCSUPTO85 la hipótesis nula que establece que estas variables son independientes del abandono de la producción láctea puede ser rechazada con un nivel de significación $p<0,1$. Los resultados indican que el abandono de la leche está relacionado positivamente con la edad del titular $(p<0,001)$ y negativamente con la superficie total de la explotación en $1985(p<0,05)$, con la producción de leche por vaca en 1985 $(p<0,05)$, con la superficie media de las parcelas en $1985(p<0,1)$, con la existencia de sucesión en la explotación $(p<0,05)$ y con el número de personas de la familia que trabajan fuera $(\mathrm{p}<0,1)$. No se encontró una relación significativa entre las variables CON y VACSUPTO85 y el abandono $(p>0,01)$. Por lo que se refiere a la variable CON, la explicación de este resultado se puede deber a que la política de concentración parcelaria en sí misma, no provoca cambios estructurales de las dimensiones de las explotaciones, por lo cual, su efecto no parece ser tan determinante en el abandono. Sin embargo, y de forma indirecta, observamos que aquellas explotaciones con mayores tamaños medios de fincas (que en muchas ocasiones son resultado directo de la aplicación de la concentración parcelaria) tienen una menor probabilidad de abandonar.

${ }^{8}$ En este sentido Alberdi (2001) señala que la progresiva aplicación de la Directiva Europea 92/46 $\mathrm{CE}$, especialmente en lo relativo a la sanidad de la cabaña y al contenido de gérmenes y células somáticas de la leche producida, ha sido uno de los factores que más ha influido en el cese de la actividad lechera en los últimos años. Según este autor, el incumplimiento de esa normativa se tradujo en penalizaciones al productor sobre el precio de la leche, que llevaron al desánimo y abandono de la actividad, lo cual además se vio favorecido por una interesante prima a cobrar por la venta de derechos de cuota. En definitiva las explotaciones pequeñas y poco modernizadas se van quedando atrás hasta que abandonan.

9 A partir de los años 90 se ha ido intensificando cada vez más la producción láctea, con la utilización masiva por parte de las explotaciones lácteas del aprovisionamiento exterior de piensos para la alimentación del ganado. Estos alimentos se destinan principalmente al lote en producción, mientras las novillas se alimentan con silo y heno. Esto facilita el mantenimiento de la producción en las explotaciones con un tamaño mínimo, donde la cabaña pasa a estar estabulada permanentemente no estando condicionado ya el aumento de la cabaña al aumento de la superficie agrícola utilizada. 


\section{TABLA 4}

Parámetros estimados y odds ratio del modelo de regresión logística para el paso de las explotaciones de leche a carne $(n=458$ explotaciones $)$

\begin{tabular}{|c|c|c|c|c|c|c|}
\hline $\begin{array}{c}\text { Variables } \\
\text { Explicativas }\end{array}$ & Coeficiente & $\begin{array}{c}\text { Error Estandar } \\
\beta\end{array}$ & $\begin{array}{c}\text { Wald } \\
\chi^{2}\end{array}$ & $\begin{array}{c}\text { Grados } \\
\text { libertad }\end{array}$ & $\mathbf{P}$ & $\begin{array}{c}\text { Odds } \\
(95 \% \text { IC) }\end{array}$ \\
\hline Constante & $-1,514$ & 0,793 & 3,644 & & & \\
\hline $\mathrm{CON}$ & 0,471 & 0,400 & 1,387 & 1 & 0,239 & $1,60(0,73-3,50)$ \\
\hline SUPTO85 & $-0,084$ & 0,041 & 4,144 & 1 & 0,042 & $0,91(0,84-0,99)$ \\
\hline VCSUPTO85 & 0,260 & 0,215 & 1,466 & 1 & 0,226 & $1,29(0,85-1,97)$ \\
\hline PLVAC85 & $-0,000$ & 0,000 & 3,923 & 1 & 0,048 & $1,00(0,99-1,00)$ \\
\hline SMPT85 & $-0,788$ & 0,406 & 3,758 & 1 & 0,053 & $0,45(0,20-1,00)$ \\
\hline IDATITU99 & 2,133 & 0,473 & 20,341 & 1 & 0,000 & $8,43(3,34-21,3)$ \\
\hline SUC99 & $-2,728$ & 1,041 & 6,867 & 1 & 0,009 & $0,06(0,00-0,50)$ \\
\hline FORA99 & $-0,463$ & 0,252 & 3,372 & 1 & 0,056 & $0,62(0,38-1,03)$ \\
\hline \multicolumn{3}{|c|}{ Test } & $x^{2}$ & $\begin{array}{r}\text { Grados } \\
\text { libertad }\end{array}$ & $\mathbf{P}$ & \\
\hline \multicolumn{7}{|c|}{ Evaluación de Modelo } \\
\hline \multicolumn{3}{|c|}{ Test de Wald } & 98,158 & 8 & 0,000 & \\
\hline \multicolumn{7}{|c|}{ Goodness-of-fit-test } \\
\hline \multicolumn{3}{|c|}{ Hosmer \& Lemeshow } & 8,236 & 8 & 0,411 & \\
\hline \multicolumn{3}{|c|}{$\%$ de casos clasificados correctamente } & $91,3 \%$ & & & \\
\hline
\end{tabular}

En nuestro estudio los odds ratio (OR) mayores que 1 significan incrementos en la probabilidad de que una explotación abandone. En la tabla 4 se muestran los OR con sus respectivos $95 \% \mathrm{IC}^{10}$. La edad del titular aumenta las probabilidades de abandonar $(\mathrm{OR}=8,43$; 95\% IC $=3,34-21,3)$. Sin embargo, la superficie total de la explotación en $1985(\mathrm{OR}=0,9195 \% \mathrm{IC}=0,84-0,99)$, la producción media por vaca en $1985(\mathrm{OR}=1,00$ 95\% IC=0,99-1,00), la superficie media de las parcelas al inicio del periodo $(\mathrm{OR}=0,45 ; 95 \%$ IC $=0,20-1,00)$, la sucesión en la explotación $(\mathrm{OR}=0,06$; $95 \%$ IC $=0,00-0,50)$ y el número de personas trabajando fuera $(\mathrm{OR}=0,62 ; \mathrm{IC}=$ 0,38-1,03) disminuyen las probabilidades de abandonar.

Para comprobar la significación global del modelo, y si el modelo calculado se ajusta bien a los datos usados para estimarlo, utilizamos Wald test que toma un valor de 98,15 con un $p=0,000$, lo que significa que el modelo calculado es más efectivo que el modelo nulo (sólo la constante). Mediante la prueba de Hosmer-Lemeshow comprobamos la calidad de predicción: el valor obtenido no es significativo $(=8,236$ $p=0,411)$, lo que sugiere que la predicción del modelo es adecuada. En otras palabras, la hipótesis nula de una buena predicción del modelo es aceptada. De hecho, el modelo predice correctamente el 91,3\% de los casos.

${ }^{10}$ El significado del limite máximo y mínimo de los $95 \%$ IC es que podemos afirmar con un $95 \%$ de seguridad que los odds ratios verdaderos estarán dentro de ese intervalo. Cuando el intervalo es amplio la precisión será más baja. 


\section{Conclusiones}

En este trabajo se han analizado las características productivas y familiares de las explotaciones que pasan de leche a carne en Galicia, con el objetivo de determinar los factores que influyen en ese proceso. Los resultados obtenidos permiten aumentar nuestro conocimiento sobre el papel que juega la edad del titular, la sucesión, la base productiva, la intensificación y el empleo extra-agrario en el cambio de orientación leche-carne. Además, de estos resultados se deriva que existe una gran similitud entre los factores que influyen tradicionalmente en el abandono de la agricultura y los que aparecen relacionados con el cambio de orientación productiva de la leche a la carne. No obstante, la comprobación de que el paso a carne es una etapa intermedia en el proceso de abandono hubiera requerido disponer en la muestra de explotaciones que abandonaron definitivamente la actividad agraria, para así compararlos con los obtenidos en este trabajo y comprobar si estas tenían características similares a las que pasaron de leche a carne.

Como hemos visto, la probabilidad de cambiar de orientación aumenta en las unidades donde el titular alcanza una edad elevada y disminuye en aquéllas que eran más grandes (mayor superficie al inicio del período), más productivas (mayor producción de leche por vaca en 1985), con mejor estructura de su base territorial (mayor tamaño de la parcela en 1985) con sucesor y con un mayor número de miembros de la familia trabajando fuera de la explotación. Finalmente, no se encuentra una relación significativa entre la concentración parcelaria y el abandono de la producción láctea, lo que viene a corroborar las reservas expresadas en otros estudios sobre el impacto real de esta política tal como se ha venido aplicando en Galicia. Sin embargo, debemos matizar que a su vez observamos que aquellas explotaciones con mayores tamaños medios de fincas (que en muchas ocasiones son resultado directo de la aplicación de la concentración parcelaria) tienen menos probabilidad de abandono. Este resultado implica que aunque la concentración parcelaria tal como es planteada en la actualidad no es una panacea para reducir el abandono de la agricultura, sus efectos indirectos no son del todo despreciables. Por ello, y para maximizar los efectos derivados de su aplicación, cabría introducir una serie de reformas de la misma, encaminadas a incrementar su efectividad.

\section{Bibliografía}

Alario Trigueros, M. (1991). Significado espacial y socioeconómico de la concentración parcelaria en Castilla León, Serie Estudios, n. ${ }^{\circ}$ 60, MAPA, Madrid.

Alberdi Collantes, J.C. (2001). «De leche a carne: hacia el abandono de la actividad agraria», Revista Española de Estudios Agro-Sociales y Pesqueros, 193:57-86.

Baur, P. (2000). «The magic growth barrier for Swiss farms». XXIV Conference of Agricultural Economics, August 13-18, 2000, Berlin, Alemania.

CIEF-IDEGA (varios años): A economía galega. Informe ... (anual). Fundación Caixa Galicia, A Coruña. 
Crecente, R., Álvarez, C. y Fra, U. (2002). «Economic, social and enviromental impact of land consolidation in Galicia». Land Use policy, 19:135-147.

De la Riba Fernández, J. (1990). «La política de concentración parcelaria en Aragón». Revista de Estudios Agro-Sociales, 151:191-236.

De Los Ríos Carmenado, I. (1996). La concentración parcelaria: concepto, análisis temporal de sus efectos y propuesta de un metamodelo basado en el aprendizaje social. Tesis Doctoral. Escuela Técnica Superior de Ingenieros Agrónomos. Universidad Politécnica de Madrid.

Fernández García, F., Maceda, A. y Quirós, F. (1992a). «Ordenación rural y concentración parcelaria en Asturias», en: El medio rural español. Cultura paisaje y naturaleza. Homenaje a don Ángel Cabo Alonso, volumen II, pp. 845-850. Ediciones Universidad de Salamanca.

Fernández García, F., Maceda, A. y Quirós, F. (1992b). «Notas sobre la concentración parcelaria en Asturias», en: El medio rural español. Cultura paisaje y naturaleza. Homenaje a don Ángel Cabo Alonso, volumen II, pp. 851-860. Ediciones Universidad de Salamanca.

Gale, H.F. (1990). «Econometric analysis of farmer participation in the dairy termination program in North Carolina and Virginia». Southern Journal of Agricultural Economics, julio 1990, 123-131.

Gasson, R., Crow, G., Errington, A., Hutson, J., Marsden, T. y Winter, D. (1995). «The Farm as a Family Business: A Review.» Reprint from the Journal of Agricultural Economics (1988) in: Peters, G.H. (Ed.), Agricultural Economics, International Library of Critical Writings in Economics. Aldershot: Edward Elgar Publishing Limited, pp. 3-43.

Glauben, T.; Hendrik, T.; Weiss, C. (2002). Intergenerational succession on family farms: evidence from survey data, Working Paper EWP 0202, Department of Food Economics and Consumption Studies, University of Kiel.

Glauben, T., Hendrik, T. y Weiss, C. (2003a). «Farm Exits: Evidence from Census Data», contributed paper, 77 AES Annual Conference, University of Plymouth, Seale-Hayne Campus, 11-13 April 2003.

Glauben, T., Hendrik, T. y Weiss, C. (2003b). Agriculture on the move: exploring regional differences in farm exits rates. Working Paper EWP 0308, Department of Food Economics and Consumption Studies, University of Kiel.

Goetz, S. y Debertin, D. (2001). «Why Farmers Quit: A County-Level Analysis». American Journal of Agricultural Economics, 83:1010-1023.

Greene, W. H. (2003). Análisis Econométrico. Quinta edición. Prentice Hall International (UK), New Jersey.

Hosmer, D. y Lemenshow, S. (1989). Applied Logistic Regression. John Wiley \& Sons.

Jackson-Smith, D. y Barham, B. (2000). The Changing face of Wisconsin Dairy Farms: a summary of PATS' research on structural change in 1990s. PATS research report $n .{ }^{\circ} 7$, August.

Kimhi, A. (1994). «Optimal timing of farm transferral from parent to child». American Journal of Agricultural Economics, 76:228-236.

Kimhi, A. y Bollman, R. (1999). «Family farm in Canada and Israel: the case of farm exits». Agricultural Economics, 21:69-79.

Kimhi, A. (2000). «Is part-time farming really a step in the way out of agriculture?». American Journal of Agricultural Economics, 82:38-48.

Lieberman, M. (1990). «Exit from declining industries: "shakeout" or "stakeout"?». RAND Journal of Economics, 21:538-554.

Liss, C.C. (1987). «Evolución y estado actual de la concentración parcelaria en España». Revista de Estudios Agro-Sociales, 139:31-66 (Madrid). 
López Garrido, C. (1993). «A produçom de carne de vacum na Galiza». Análise Empresarial, 18:61-68.

López Iglesias, E. (1996). Movilidad de la tierra y dinámica de las estructuras agrarias en Galicia. MAPA, Madrid.

López Iglesias, E. (1998). «El sector agrario en Galicia: alcance y límites de su reestructuración en la última década», en: Molina, M.; Muñoz, C. y Ruiz-Maya, L. (Coord.) El sector agrario. Análisis desde las Comunidades Autónomas. MAPA, Madrid.

MAPA (1978). Tipificación de las comarcas agrarias españolas. MAPA, Secretaría General Técnica, Madrid.

MAPA (2000). Anuario de estadistica agroalimentaria 2000. MAPA, Madrid.

MAPA (2005). Evolución de las Macromagnitudes Agrarias Regionales 1990-2000 (disponible en la web www.mapya.es).

Neyman, J. (1934). «On the two different aspects of the representative method: the method of stratified sampling and the method of purposive selection». Journal of the Royal Statistical Society, 97:558-606.

Paniagua, A. (2000). «Analysis of the evolution of farmers' early retirement policy in Spain. The case of Castille and Leon». Land Use Policy, 17:113-120.

Pfeffer, M. J. (1989). «Part-time farming and the stability of family farms in the Federal Republic of Germany». European Review of Agricultural Economics, 16(4):425-444.

Pietola, K., Väre, M. y Oude Lansink, A. (2003). «Timing and type of exist form farming: farmers' early retirement programmes in Finland». European Review of Agricultural Economics, 30:99-116.

Ribas, A. (2004). A incidencia da concentración parcelaria na dinámica das explotacións agrarias. Análise para as explotacións bovinas da provincia de A Coruña. Tesis Doctoral inédita. Universidade de Santiago de Compostela.

SAS (1995). Logistic Regression Examples, Using the SAS System. SAS Institute Inc.

Sineiro, F., López Iglesias, E., Lorenzana, R. y Valdés, B. (2005). «La tipología de las explotaciones en función de su viabilidad económica y demográfica; aplicación a las explotaciones de bovino en Galicia». Economía Agraria y Recursos Naturales, 4 (8).

Stiglbauer, A. y Weiss, C. (2000). «Family and non-family succession in the Upper-Austrian farm sector». Cahiers d'économie er sociologie rurales, 54:6-24.

Swidinsky, M., Howard, W. y Weersink, A. (1998). Off-farm work by census-farm operators: an overview of structure and mobility patterns. Working Paper, 38. Statistics Canada Agriculture División.

Van Huylenbroek, G. y Martens, L. (1996). «Evaluation of land consolidation projets (LCPs): a multidisciplinary approach». Journal of Rural Studies, 12 (3):297-310.

Weiss, C. (1996). Exits from a declining sector: econometric evidence from a panel of Upper Austrian farms 1980-90. Working Paper No. 9601, Johannes Kepler University of Linz.

Weiss, C. R. (1997). «Do they ever come back again? The symmetry and reversibility of offfarm employment». European Review of Agricultural Economics, 24(1):65-84.

Weiss, C.R. (1999). «Farm growth and survival: econometric evidence for individual farms in Upper Austria». American Journal of Agricultural Economics, 81 (1):103-116. 